\title{
Evolutionary simulation modelling clarifies interactions between parallel adaptive processes
}

\author{
Seth Bullock and Jason Noble \\ Center for Adaptive Behavior and Cognition \\ Max-Planck-Institut für Bildungsforschung \\ Lentzeallee 94, D-14195 Berlin, GERMANY \\ bullock, noble@mpib-berlin.mpg.de \\ http://www-abc.mpib-berlin.mpg.de/
}

\begin{abstract}
The teleological language in the target article is ill-advised, as it obscures the question of whether ecological and cultural inheritances are directed or random. The authors present a very broad palette of explanatory possibilities; evolutionary simulation models could help narrow down the processes important in a particular case. Examples of such models are offered in the areas of language change and the Baldwin effect.
\end{abstract}

\section{Commentary}

The central theoretical message of the target article is that, through modifying their environment, organisms affect the selection pressures acting on them. The extent to which previous students of evolution have been unaware of this interaction is debatable; for example, orthodox models of coevolution address changes in selection pressure brought about by the evolution of new traits. The novel contribution made in this article is simply to note that, to the extent that these new traits affect the environment, they may have additional effects on selection which may persist for longer than the lifetime of an individual organism. Nevertheless, the authors are to be commended for 
outlining a theoretical framework that makes these matters explicit.

We were somewhat alarmed by the authors' pervasive use of teleological language in describing the processes of "niche construction". Whilst evolution is clearly an undirected process, and ontogenetic development (including learning) is equally clearly goal-directed, the status of some nascent, intermediate adaptive level is far less straightforward. In their use of terms such as "counteractive niche construction", do the authors mean to suggest that cultural or ecological inheritance should be considered to be purposive after the fashion of individual learning? If so, must there have been natural selection for the ability to construct niches in the same way that there has been natural selection for the ability to learn? The issue is not merely a linguistic one, since we know that very different dynamics are to be expected from directed as opposed to non-directed adaptive systems. Consider that, since mutations in general are deleterious, niches constructed due to genetic mutation (e.g., web building by spiders) will be rare success stories among many failures. However, since the fitness consequences of novel learned behaviours may be distributed very differently to those of genetic mutations, and will depend on the specific learning mechanism involved, the success rate of niches constructed through learning (e.g., the learned use of a grubbing tool by woodpecker finches) will differ accordingly.

The interaction between genetic evolution, learning, and intervening adaptive processes will turn on specific facts about genetic constraints, learning biases, and the environment of the organisms involved. Although we appreciate the value of the target article in introducing such a wide range of explanatory possibilities, individual cases demand individual explanations. A move in this direction has been achieved by the emerging field of evolutionary simulation modelling (see Belew \& Mitchell, 1996, for examples). This paradigm employs models that simulate the dynamic evolution of a population 
of agents subject to some adaptive process in order to test theories concerning analogous natural systems. These models stand somewhere between the abstraction of mathematical work and the complexity of the real world. They could be used to expand upon the theoretical framework of the target article, by examining the relative importance of different processes (e.g., genetic evolution, learning, cultural inheritance, and niche construction) in specific cases.

In some evolutionary simulations, there is little room for what Laland et al. refer to as "ecological inheritance", as the environment is wiped clean for each new generation of simulated organisms. However, in other simulations the behaviour of one generation does affect the selection pressures impinging on the next, either because new organisms continuously arrive in an established population, or because the behaviour of adults is recorded in some way and used as part of the environment for their children. An example of the latter is Kirby \& Hurford's 1997 model of language evolution. New-born organisms must learn a grammar from a set of utterances provided by the parental generation. Thus the ecological legacy is not the physical environment but the linguistic one: a new organism is born into a world of speakers. Kirby \& Hurford use their model to challenge Chomskyan orthodoxy, and show that the "evolution" of the language itself, towards greater parsability, is actually prior to the genetic fixation of the grammatical structure. They have used their simulation to go beyond the general observation that genetic and linguistic inheritances may interact, and shown how they may be expected to do so.

The logic of the target article is based on mathematical models outlined elsewhere (Laland et al., 1996; Odling-Smee et al., 1996). The conclusions that the authors have drawn are no doubt sound, but such mathematical modelling can conceal many implicit assumptions. Another virtue of evolutionary simulations is that, like models in artificial intelligence, they force their creator 
to be explicit in every detail. Consider the work of Mayley (1996) on the Baldwin effect - this effect is very close to the concerns of the target article as it involves an interaction between learning and genetic evolution. Mayley uses an evolutionary simulation to demonstrate that the conditions under which the Baldwin effect will result in the genetic fixation of a learned trait are not straightforward. The costs as well as the benefits of learning, and the correlation between genotypic space and the space of behavioural strategies, must be taken into account. Earlier authors had certainly looked at the costs and benefits of learning, but had tended to assume that genotypic and phenotypic space were in perfect correspondence. Such complexities are often glossed over in the kind of abstract mathematical model constructed by Laland et al. Yet they remain of great importance to those interested in understanding specific evolved phenomena.

Finally, at one point the authors note enthusiastically that, given their framework, "the suite of hypotheses about... evolutionary change is considerably enlarged." We would remind the reader that an essential task in science is to reduce the number of hypotheses that are plausible with respect to a given phenomenon; we believe that building evolutionary simulation models can help to achieve that. 


\section{References}

Belew, R. \& Mitchell, M. (eds.) (1996). Adaptive Individuals in Evolving

Populations: Models and Algorithms. Addison-Wesley, Reading, MA.

Kirby, S. \& Hurford, J. (1997). Learning, culture and evolution in the origin of linguistic constraints. In P. Husbands \& I. Harvey (eds.), Proceedings of the Fourth European Conference on Artificial Life (ECAL'97), pp. 493-502, MIT Press / Bradford Books, Cambridge, MA.

Laland, K. N., Odling-Smee, F. J., \& Feldman, M. W. (1996). On the evolutionary consequences of niche construction. Journal of Evolutionary Biology, 9, 293-316.

Mayley, G. (1996). Landscapes, learning costs and genetic assimilation. Evolutionary Computation, 4(3), 213-234.

Odling-Smee, F. J., Laland, K. N., \& Feldman, M. W. (1996). Niche construction. American Naturalist, 147(4), 641-648. 